\title{
Drift-Wave Instabilities Modified by Parallel and Perpendicular Flow Velocity Shears in Magnetized Plasmas
}

\author{
Toshiro KANEKO, Shuichi TAMURA, Atsushi ITO $^{1)}$ and Rikizo HATAKEYAMA \\ Department of Electronic Engineering, Tohoku University, Sendai 980-8579, Japan \\ ${ }^{1)}$ National Institute for Fusion Science, Toki 509-5292, Japan
}

(Received 7 December 2007 / Accepted 29 January 2008)

\begin{abstract}
Plasma flow velocity shears parallel and perpendicular to magnetic field lines are independently controlled and superimposed by a modified plasma-synthesis method with concentrically three-segmented electron and ion emitters. The fluctuation amplitude of a drift wave with an azimuthal mode number $m=3$ is observed to increase with increasing parallel shear strength in the absence of a perpendicular shear. When the perpendicular shear is superimposed on the parallel shear, the drift wave with $m=3$ is found to transform into that with $m=2$. Furthermore, the parallel shear strength required for excitation of the drift wave increases with a decrease in the azimuthal mode number. Based on these results, superposition of the parallel and perpendicular shears can affect characteristics of the drift wave through variation of the azimuthal mode number. These phenomena can be verified by theoretical calculations of the growth rate of the drift wave using an eigenmode analysis.
\end{abstract}

(C) 2008 The Japan Society of Plasma Science and Nuclear Fusion Research

Keywords: plasma flow velocity shear, drift wave, azimuthal mode number, plasma-synthesis method

DOI: $10.1585 /$ pfr.3.S1011

\section{Introduction}

Plasma flows and their velocity shears in magnetized plasmas have attracted much attention not only in fusion oriented plasmas but also in space plasmas, because the ion flow velocity shear parallel to the magnetic field lines has been reported to enhance the ion-acoustic $[1,2]$, ioncyclotron [3,4], and drift-wave [5, 6] instabilities, while the perpendicular flow velocity shear has been confirmed to regulate not only the drift-wave but also ion-cyclotron instabilities, independent of the sign of the shear [7]. In order to clarify the mechanisms of excitation and suppression of these instabilities in real fusion and space plasmas, it is necessary to realize the controlled superposition of the parallel and perpendicular flow shears in magnetized plasmas $[8,9]$.

Thus, the aim of the present work is to independently control and superimpose the parallel and perpendicular flow shears in a basic plasma device with concentrically three-segmented electron and ion emitters [10], and to perform laboratory experiments on the drift-wave instability, which is excited and suppressed by superimposed flow shears in collisionless magnetized plasmas.

\section{Experimental Setup}

Experiments are performed in the $\mathrm{Q}_{\mathrm{T}}$-Upgrade machine of Tohoku University. We attempt to modify a plasma-synthesis method using an electron $\left(\mathrm{e}^{-}\right)$emitter with a $10-\mathrm{cm}$-diameter tungsten $(\mathrm{W})$ plate and a potassium ion $\left(\mathrm{K}^{+}\right)$emitter with another $\mathrm{W}$ plate, which are

author'se-mail: kaneko@ecei.tohoku.ac.jp

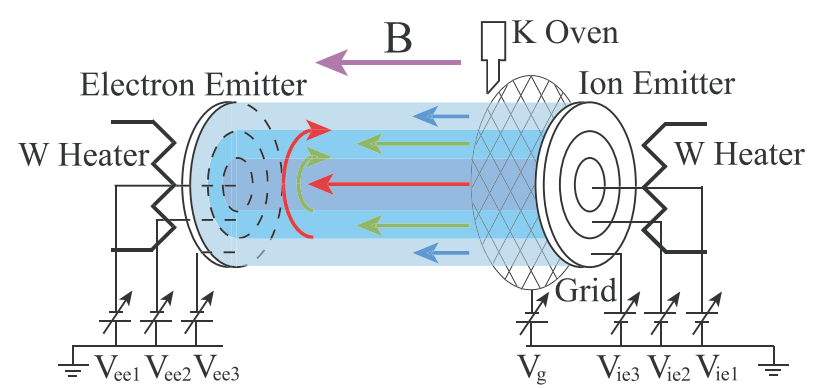

Fig. 1 Schematic of experimental setup.

oppositely located at the machine ends as shown in Fig. 1. A collisionless plasma is produced when surface-ionized potassium ions and thermionic electrons are generated using the spatially separated ion and electron emitters, respectively, and are synthesized in the region between these emitters. A negatively biased stainless (SUS) grid, the voltage of which is typically $V_{g}=-60 \mathrm{~V}$, is installed at a distance of $10 \mathrm{~cm}$ from the ion emitter surface. Since the grid reflects the electrons flowing from the electron emitter side, the electron velocity distribution function parallel to the magnetic fields are considered Maxwellian.

Both the emitters are segmented concentrically into three sections with the outer diameters of 2 (first electrode), 5.2 (second electrode), and $10 \mathrm{~cm}$ (third electrode), each of which is electrically isolated. When each section of the electron emitter is individually biased, a radially varying plasma potential, i.e., radial electric field is expected to be generated even in a fully ionized collisionless plasma. This electric field causes $E \times B$ flows and flow shears per- 
pendicular to the magnetic-field lines. Voltages applied to the electrodes set in order from the center to the outside are defined as $V_{\text {ee1 }}, V_{\text {ee2}}$, and $V_{\text {ee3 }}$, respectively. On the other hand, a parallel $\mathrm{K}^{+}$flow with radially varying energy, i.e., the parallel $\mathrm{K}^{+}$flow shear, is generated when each section of the segmented ion emitter is individually biased $\left(V_{\mathrm{ie} 1}, V_{\mathrm{ie} 2}, V_{\mathrm{ie} 3}\right)$ at a positive value above the plasma potential, which is determined by the bias voltage of the electron emitter. Therefore, these parallel and perpendicular $\mathrm{K}^{+}$flow velocity shears can be superimposed by controlling the bias voltage of the ion and electron emitters independently. Here, $V_{\text {ee } 3}$ and $V_{\text {ie3 }}$ are always maintained at $0 \mathrm{~V}$. A small radially movable Langmuir probe and an electrostatic energy analyzer are used to measure radial profiles of plasma parameters and ion energy distribution functions parallel to the magnetic fields, respectively. Under our conditions, the plasma density is $10^{9} \mathrm{~cm}^{-3}$, the electron temperature is $0.2 \mathrm{eV}$, and the ion temperature is almost the same as the electron temperature. The background gas pressure is less than $10^{-6}$ Torr.

\section{Experimental Results}

First, we demonstrate the independent control of the parallel and perpendicular $\mathrm{K}^{+}$flow velocity shears and superposition of these shears. Figure 2 shows the plasma potential $\phi$ (closed circles) and the $\mathrm{K}^{+}$flow energy $\mathrm{E}_{\mathrm{K}^{+}}$(open circles) at the radial center $r=0 \mathrm{~cm}$ of the plasma column as functions of $V_{\text {ie1 }}$ and/or $V_{\text {ee } 1}$, where $V_{\text {ie2 }}$ and $V_{\text {ee2 }}$ are fixed at 5.0 and $-2.0 \mathrm{~V}$, respectively. When $V_{\text {iel }}$ is varied at constant $V_{\text {ee } 1}=-2.2 \mathrm{~V}$ (Fig. 2 (a)), the $\mathrm{K}^{+}$flow energy is found to increase in proportion to $V_{\mathrm{ie} 1}$, while the plasma potential is almost constant at $\phi=-5.5 \mathrm{~V}$. Since the $\mathrm{K}^{+}$ flow energy and the plasma potential in the second electrode region are confirmed to have constant values of $7 \mathrm{eV}$ and $-5.5 \mathrm{~V}$, respectively, only the parallel flow velocity shear can be generated in the boundary region between the first and second electrodes by varying $V_{\text {ie } 1}$.

On the other hand, when $V_{\mathrm{ie} 1}$ and $V_{\mathrm{ee} 1}$ are varied simultaneously maintaining the bias-voltage difference $V_{\text {ie } 1}-V_{\text {ee } 1}$ constant (Fig. 2 (b)), the $\mathrm{K}^{+}$flow energy does not change, while the plasma potential is found to increase in proportion to $V_{\mathrm{ee} 1}$. This result denotes that the parallel shear does not change if $V_{\text {ie } 1}-V_{\text {ee } 1}$ is constant, and the radial plasma potential difference, i.e., the perpendicular flow velocity shear can be controlled by the bias voltages of the electron emitter. Since the parallel and perpendicular shears can be controlled independently, we attempt to superimpose these shears.

Figure 2 (c) presents the plasma potential and $\mathrm{K}^{+}$flow energy as a function of $V_{\mathrm{ee} 1}$ at constant $V_{\mathrm{ie} 1}=5 \mathrm{~V}$. In this case, the plasma potential and $\mathrm{K}^{+}$flow energy varies with $V_{\text {eel }}$, because the bias-voltage difference $V_{\text {ie } 1}-V_{\text {ee1 }}$ decreases with an increase in $V_{\text {ee } 1}$ for fixed $V_{\text {iel } 1}$. Based on these results, the superposition of the parallel and perpendicular flow velocity shears is realized by controlling $V_{\text {iel }}$
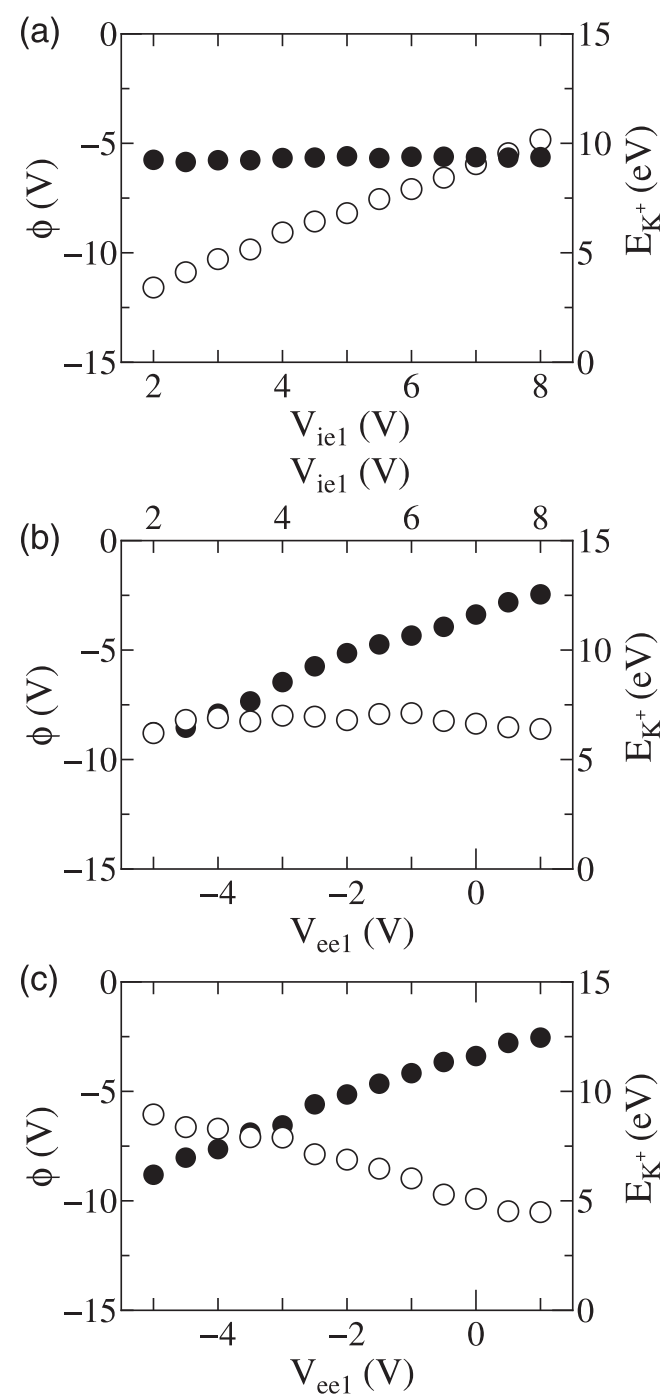

Fig. 2 Plasma potential $\phi$ (closed circles) and $\mathrm{K}^{+}$flow energy $E_{\mathrm{K}^{+}}$(open circles) as functions of (a) $V_{\mathrm{iel}}$, (b) $V_{\mathrm{iel}}$ and $V_{\mathrm{ee} 1}$, and (c) $V_{\mathrm{ee} 1} \cdot r=0 \mathrm{~cm}, V_{\mathrm{ee} 2}=5.0 \mathrm{~V}$, and $V_{\mathrm{ee} 2}=$ $-2.0 \mathrm{~V}$.

and $V_{\text {eel }}$ simultaneously. These parallel and perpendicular shears are found to cause several types of low-frequency instabilities. Here, we concentrate on a drift-wave instability excited in the density gradient region around $r=1.0$ $1.5 \mathrm{~cm}$.

Figure 3 (a) shows a contour view of normalized fluctuation amplitudes $\tilde{I}_{\mathrm{es}} / \bar{I}_{\mathrm{es}}$ obtained from frequency spectra of an electron saturation current $I_{\mathrm{es}}$ of the probe as functions of $V_{\mathrm{ie} 1}$ and $V_{\mathrm{ee} 1}$ for $V_{\mathrm{ie} 2}=1.0 \mathrm{~V}$ and $V_{\text {ee2 }}=-2.0 \mathrm{~V}$. Schematic model of the parallel and perpendicular shears introduction is shown in Fig. $3(\mathrm{~b})$, where black arrows present at the ordinate axis indicate the parallel ion flow velocity and solid curves present at abscissa axis indicate the radial potential profiles, which are controlled by $V_{\mathrm{ie} 1}$ and $V_{\mathrm{ee} 1}$, respectively, corresponding to the variation of the parallel and perpendicular flow velocity shears. Here, horizontal and vertical dotted lines in Fig. 3 denote the situations in 


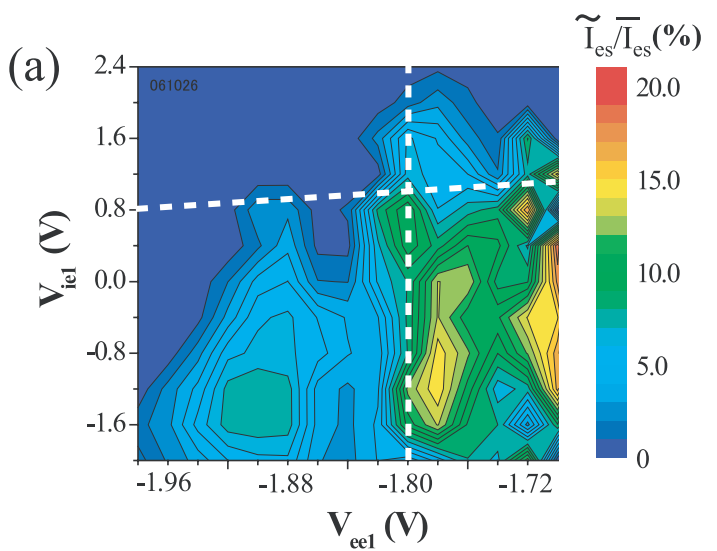

(b)

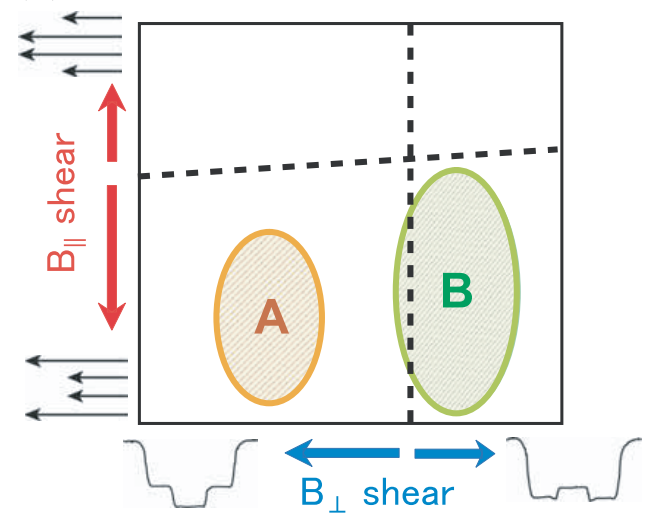

Fig. 3 Contour views of normalized fluctuation amplitudes as functions of $V_{\mathrm{ie} 1}$ and $V_{\mathrm{ee} 1} . r=1.5 \mathrm{~cm}, V_{\mathrm{ie} 2}=1.0 \mathrm{~V}$, and $V_{\mathrm{ee} 2}=-2.0 \mathrm{~V}$.

the absence of the parallel and perpendicular shears, respectively, which are confirmed by the actual measurements of the ion flow energy and space potential.

In the case that the perpendicular shear is not generated at $V_{\text {ee1 }}=-1.8 \mathrm{~V}$, the fluctuation amplitude of the drift-wave instability is observed to increase with increasing the parallel shear strength by changing $V_{\text {ie } 1}$ from $1.0 \mathrm{~V}$ to negative values, but the instability is found to be stabilized gradually when the shear strength exceeds the critical value. The destabilizing and stabilizing mechanisms are well explained by the plasma kinetic theory including the effect of radial density gradient [5]. When the perpendicular shear is superimposed on the parallel shear, the drift wave excited by the parallel shear is found to be suppressed by the perpendicular shear independently of the sign of the perpendicular shear. Furthermore, we can observe two characteristic fluctuation peaks depending on the perpendicular shear strength as presented in the contour views (Fig. 3 (a)), which are defined as fluctuations A and B as described in the schematic model (Fig. 3(b)).

To readily identify the azimuthal component of each fluctuation's wavevector, we measure 2-dimensional $(x, y)$ profiles of fluctuation phase in the plasma-column cross section. The phase is measured with reference to a spatially fixed Langmuir probe located at an axial distance
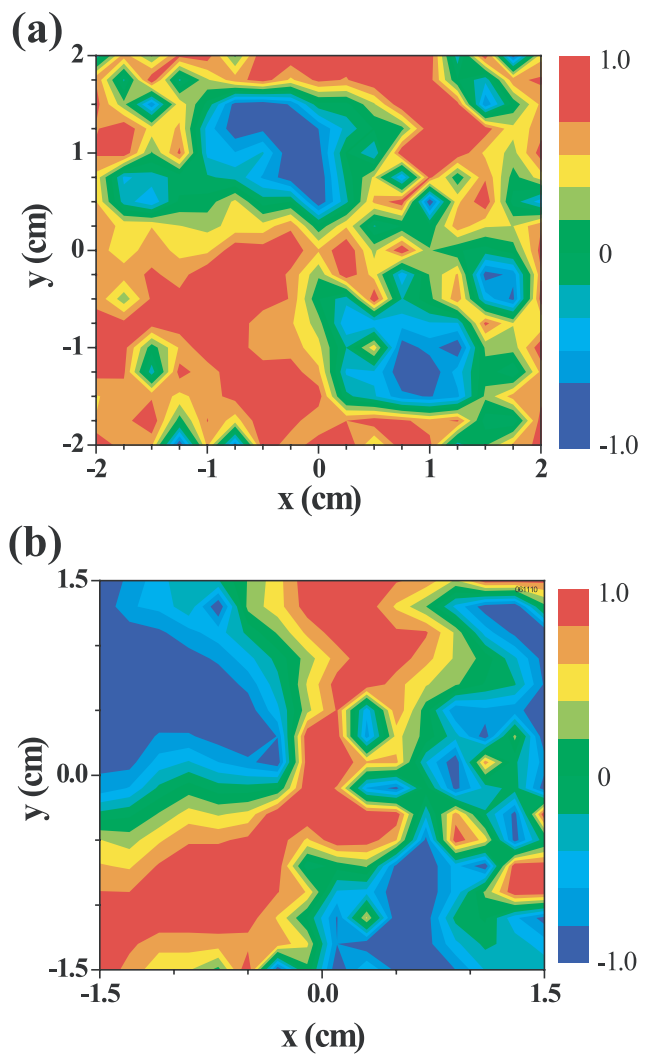

Fig. 4 2-dimensional profile of fluctuation phase $\theta$ which is plotted as $\sin \theta$ for (a) fluctuation $\mathrm{A}\left(V_{\mathrm{ee} 1} \simeq-1.9 \mathrm{~V}\right)$ and $(\mathrm{b})$ fluctuation $\mathrm{B}\left(V_{\mathrm{ee} 1} \simeq-1.8 \mathrm{~V}\right) . V_{\mathrm{ie} 1}=-1 \mathrm{~V}, V_{\mathrm{ie} 2}=1 \mathrm{~V}$, and $V_{\mathrm{ee} 2}=-2 \mathrm{~V}$.

of $26 \mathrm{~cm}$ from a 2-dimensional scanning probe. Figure 4 presents 2-dimensional phase profiles for (a) fluctuation A and (b) fluctuation B. Since the phase difference $\theta$ between the 2-dimensional probe and the reference probe is plotted as $\sin \theta$, the phase of $+\pi / 2$ and $-\pi / 2$ relative to the reference probe are indicated by 1.0 (red) and -1.0 (blue), respectively. Green corresponds to a phase difference of zero and $\pi$. In the case of the small perpendicular shear strength, i.e., fluctuation B (Fig. 4 (b)), the azimuthal mode is found to be $m=3$. On the other hand, in the presence of the relatively large perpendicular shear, i.e., fluctuation A (Fig. 4 (a)), the azimuthal mode becomes $m=2$. The perpendicular shear is found to modify the azimuthal mode number depending on its strength.

For these two kinds of drift waves, we measure the dependence of fluctuation amplitudes on the parallel shear strength. Figure 5 shows normalized fluctuation amplitude $\tilde{I}_{\mathrm{es}} / \bar{I}_{\mathrm{es}}$ as a function of $V_{\mathrm{ie} 1}$ for $V_{\mathrm{ee} 1} \simeq-1.8 \mathrm{~V}$ (closed circles) and $-1.9 \mathrm{~V}$ (open circles), which are obtained from Fig. 3 (a). The dotted line in Fig. 5 denotes the bias voltage of the second electrode $V_{\text {ie2} 2}$, i.e., the absence of the parallel shear. In this experimental condition, a drift wave is excited even in the absence of the parallel shear for $V_{\text {ee1 }} \simeq-1.8 \mathrm{~V}$ because of the relatively large parallel ion flow (current) [11]. With an increase in the parallel shear 


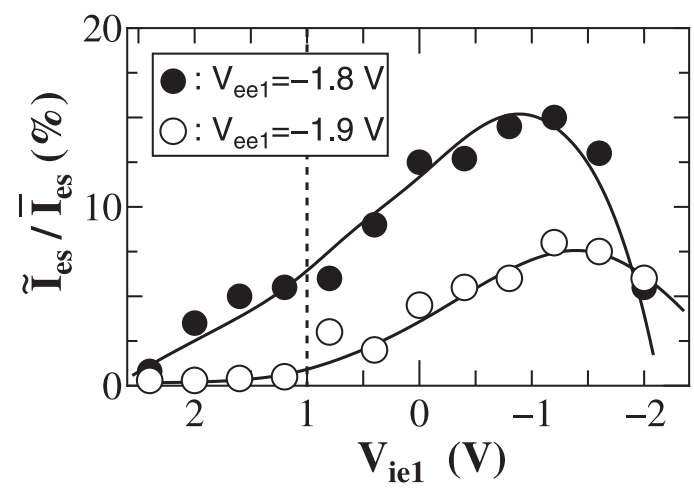

Fig. 5 Normalized fluctuation amplitudes $\tilde{I}_{\mathrm{es}} / \bar{I}_{\mathrm{es}}$ as a function of $V_{\text {ie1 }}$ for $V_{\text {ie } 2}=1 \mathrm{~V}$ at $r=-1.0 \mathrm{~cm}$.

strength, it is found that the $m=3$ mode $\left(V_{\text {ee } 1} \simeq-1.8 \mathrm{~V}\right)$ is first excited and the $m=2$ mode $\left(V_{\text {ee } 1} \simeq-1.9 \mathrm{~V}\right)$ requires the higher parallel shear strength to be excited.

These phenomena can be explained by a theoretical calculation of the growth rate of the drift wave modified by velocity shears using eigenmode analysis. We model the region near the plasma-column edge using slab geometry, in which case $\mathrm{d} v_{\mathrm{d}} / \mathrm{d} r=\mathrm{d} v_{\mathrm{d}} / \mathrm{d} x$ and $k_{\theta}=m / r=k_{y}$, in the limit of the radial position of the drift waves much larger than both the ion gyroradius and annular width of the radial profile of fluctuation amplitude. The eigenmode equation is given as

$$
\frac{\mathrm{d}^{2} \psi(x)}{\mathrm{d} x^{2}}-\frac{1+\sum \Gamma_{n}(b) F_{n \mathrm{i}}(x)+\tau\left(1+F_{0 \mathrm{e}}(x)\right)}{\sum \Gamma_{n}^{\prime}(b) F_{n \mathrm{i}}(x)} \psi(x)=0,
$$

where the details of the mathematical derivations and descriptions can be found in Ref. [12] and are not repeated here. Note that the boundary condition is $\psi( \pm \infty)=0$. Spatial profiles of the parallel and perpendicular flow velocities of the ion and electron are described as

$$
\begin{aligned}
& V_{\mathrm{i} \|}(x)=\frac{V_{\| 0}}{2} \tanh \left(\frac{x}{L_{0}}\right), \\
& V_{\mathrm{e} \|}(x)=V_{\mathrm{e} \| 0}+V_{\mathrm{i} \|}, \\
& V_{\perp}(x)=V_{\perp 0} \cosh ^{-2}\left(\frac{x}{L_{0}}\right),
\end{aligned}
$$

and the density gradient $(\mathrm{d} \ln n / \mathrm{d} x)$ is assumed to be constant. The calculation code is based on that in Ref [13].

Figure 6 presents the thresholds at which the growth rate of the drift wave becomes positive (unstable) as functions of the parallel and perpendicular flow velocity shears, where the regions above and below from the threshold lines indicate the stable and unstable conditions, respectively. The density gradient and other parameters are set to $\rho_{\mathrm{i}}(\mathrm{d} \ln n / \mathrm{d} x)=-0.01, L_{0}=10 \rho_{\mathrm{i}}, V_{\mathrm{e} \| 0}=60 v_{\mathrm{ti}}, k_{z} \rho_{\mathrm{i}}=$ $2.4 \times 10^{-3}, m_{\mathrm{i}} / m_{\mathrm{e}}=71628, T_{\mathrm{i}}=T_{\mathrm{e}}$, and $\rho_{\mathrm{i}} \equiv v_{\mathrm{ti}} / \omega_{\mathrm{ci}}$. The parameters for the calculation of the growth rate correspond well with the experimentally obtained values in this experimental condition except for the larger parallel electron drift velocity $V_{\mathrm{e} \|}$ and the smaller density gradient. The

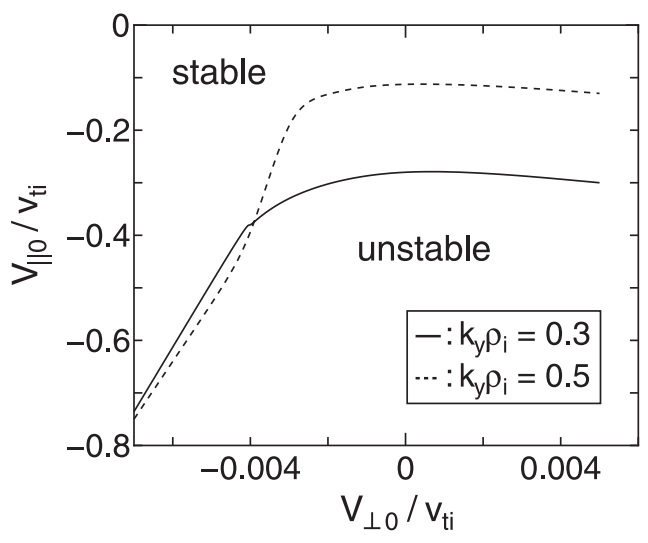

Fig. 6 Threshold of stability for the drift wave as functions of parallel and perpendicular flow velocity shears using eigenmode analysis. The superior and inferior regions from the lines mean the stable and unstable conditions, respectively.

drift wave instability is the ion acoustic wave destabilized by the density gradient, and the current driven ion acoustic instability is the wave destabilized by the electron drift velocity. In both of the instabilities, the effects of flow velocity shear act mainly through the modulation of the real frequency of the ion acoustic wave in a similar way [5,14]. Thus, the result is expected to be qualitatively insensitive to the values of the parallel electron drift velocity and density gradient.

In the case that the perpendicular shear is small, i.e., $V_{\perp 0} / v_{\mathrm{ti}} \simeq 0$, the drift wave becomes unstable with an increase in the parallel-shear strength, first at $\left|V_{\| 0} / v_{\mathrm{ti}}\right| \simeq 0.1$ for $k_{y} \rho_{\mathrm{i}}=0.5$, then at $\left|V_{\| 0} / v_{\mathrm{ti}}\right| \simeq 0.3$ for $k_{y} \rho_{\mathrm{i}}=0.3$. Here, $k_{y} \rho_{\mathrm{i}}=0.3$ and 0.5 correspond to the experimentally obtained azimuthal mode numbers $m=2$ and 3, respectively. When the perpendicular shear becomes relatively large, i.e., $V_{\perp 0} / v_{\mathrm{ti}} \simeq-0.005$, the unstable drift wave appears around $\left|V_{\| 0} / v_{\mathrm{ti}}\right| \simeq 0.5$, which is larger than that in the case of $V_{\perp 0} / v_{\mathrm{ti}} \simeq 0$. Furthermore, with an increase in the parallel shear, the drift wave becomes unstable first for $k_{y} \rho_{\mathrm{i}}=0.3$ and next for $k_{y} \rho_{\mathrm{i}}=0.5$; this tendency is opposite to that for the case of $V_{\perp 0} / v_{\mathrm{ti}} \simeq 0$.

Based on these theoretical results, it is found that the drift wave with the larger azimuthal mode number can be excited by the smaller parallel shear strength in the absence of the perpendicular shear, while the wave with the smaller azimuthal mode number is easily excited in the presence of the relatively large perpendicular shear. This explains the experimentally obtained results that the modes with $m=3$ is excited in the absence of perpendicular shear, and the mode number becomes $m=2$ for the relatively large perpendicular shear as shown in Figs. 3 and 4. The theoretical results can also verify the experimental result that a larger parallel shear is required to excite a drift wave in a larger perpendicular shear. Since the growth rate of a parallel-shear excited drift wave depends sensitively on the azimuthal mode number that depends on the perpendic- 
ular shear, the superposition of the parallel and perpendicular shears can affect the characteristics of the drift wave through the variation of the azimuthal mode number.

\section{Conclusions}

Independent control of parallel and perpendicular flow velocity shears in magnetized plasmas is realized by a modified plasma-synthesis method with segmented plasma sources. The ion flow velocity shear parallel to the magnetic-field lines is observed to destabilize the driftwave instability depending on the strength of the parallel shear. On the other hand, when the perpendicular shear is superimposed on the parallel shear, the drift wave with $m=3$ is found to change into that with $m=2$, and the instability is suppressed for strong perpendicular shears. The superposition of these shears can affect the characteristics of the drift wave through the variation of the azimuthal mode number.

\section{Acknowledgements}

The authors are indebted to $\mathrm{H}$. Ishida for his technical assistance. We also express our gratitude to Professor M. Koepke and Dr. G. Ganguli for their useful comments and discussion. This work was supported by a Grant-in-Aid for Scientific Research from the Ministry of Education, Cul- ture, Sports, Science and Technology, Japan.

[1] E. Agrimson, N. D’Angelo and R.L. Merlino, Phys. Rev. Lett. 86, 5282 (2001).

[2] C. Teodorescu, E.W. Reynolds and M.E. Koepke, Phys. Rev. Lett. 88, 185003 (2002).

[3] C. Teodorescu, E.W. Reynolds and M.E. Koepke, Phys. Rev. Lett. 89, 105001 (2002).

[4] E. Agrimson, S.-H. Kim, N. D'Angelo and R.L. Merlino, Phys. Plasmas 10, 3850 (2003).

[5] T. Kaneko, H. Tsunoyama and R. Hatakeyama, Phys. Rev. Lett. 90, 125001 (2003).

[6] T. Kaneko, E.W. Reynolds, R. Hatakeyama, and M.E. Koepke, Phys. Plasmas 12, 102106 (2005).

[7] R. Hatakeyama and T. Kaneko, Phys. Scripta T107, 200 (2004).

[8] T. Kaneko, K. Hayashi, R. Ichiki and R. Hatakeyama, Trans. Fusion Sci. Technol. 51, 103 (2007).

[9] M.E. Koepke and E.W. Reynolds, Plasma Phys. Control. Fusion 49, A145 (2007)

[10] T. Kaneko, Y. Odaka, E. Tada and R. Hatakeyama, Rev. Sci. Instrum. 73, 4218 (2002).

[11] R. Hatakeyama, M. Oertl, E. Märk and R. Schrittwieser, Phys. Fluids 23, 1774 (1980).

[12] G. Ganguli, M. J. Keskinen, H. Romero, R. Heelis, T. Moore and C. Pollock, J. Geophys. Res. 99, 8873 (1994).

[13] A. Ito and A. Hirose, Phys. Plasmas 11, 23 (2004).

[14] V.V. Gavrishchaka, S.B. Ganguli, G.I. Ganguli, Phys. Rev. Lett. 80, 728 (1998). 